\title{
Publisher Correction: Dynamic structural states of ClpB involved in its disaggregation function
}

\author{
Takayuki Uchihashi (1) 1, Yo-hei Watanabe ${ }^{2,3}$, Yosuke Nakazaki ${ }^{2,3}$, Takashi Yamasaki ${ }^{2,3}$, Hiroki Watanabe ${ }^{4}$, \\ Takahiro Maruno ${ }^{5}$, Kentaro Ishii ${ }^{6}$, Susumu Uchiyama (10 5,6, Chihong Song7, Kazuyoshi Murata7, \\ Ryota lino (i) ${ }^{8,9}$ \& Toshio Ando (1) ${ }^{10}$
}

Correction to: Nature Communications https://doi.org/10.1038/s41467-018-04587-w, published online 1 June 2018.

The original version of this Article did not acknowledge Ryota lino as a corresponding author. This has now been corrected in both the PDF and HTML versions of the Article.

Published online: 12 July 2019

\begin{abstract}
(c) Open Access This article is licensed under a Creative Commons Attribution 4.0 International License, which permits use, sharing, adaptation, distribution and reproduction in any medium or format, as long as you give appropriate credit to the original author(s) and the source, provide a link to the Creative Commons license, and indicate if changes were made. The images or other third party material in this article are included in the article's Creative Commons license, unless indicated otherwise in a credit line to the material. If material is not included in the article's Creative Commons license and your intended use is not permitted by statutory regulation or exceeds the permitted use, you will need to obtain permission directly from the copyright holder. To view a copy of this license, visit http://creativecommons.org/licenses/by/4.0/.
\end{abstract}

(C) The Author(s) 2019

\footnotetext{
${ }^{1}$ Department of Physics and Structural Biology Research Center, Nagoya University, Chikusa-ku, Nagoya 464-8602, Japan. ${ }^{2}$ Department of Biology, Faculty of Science and Engineering, Konan University, Okamoto 8-9-1, Kobe 658-8501, Japan. ${ }^{3}$ Institute for Integrative Neurobiology, Konan University, Okamoto 8 9-1, Kobe 658-8501, Japan. ${ }^{4}$ Department of Physics, College of Science and Engineering, Kanazawa University, Kanazawa 920-1192, Japan. ${ }^{5}$ Department of Biotechnology, Graduate School of Engineering, Osaka University, Osaka 565-0871, Japan. ${ }^{6}$ Exploratory Research Center on Life and Living Systems (ExCELLS), National Institutes of Natural Sciences, Okazaki, Aichi 444-8787, Japan. ${ }^{7}$ National Institute for Physiological Sciences, National Institutes of Natural Sciences, Okazaki, Aichi 444-8787, Japan. ${ }^{8}$ Institute for Molecular Science, National Institutes of Natural Sciences, Okazaki, Aichi 444-8787, Japan. ${ }^{9}$ Department of Functional Molecular Science, School of Physical Sciences, The Graduate University for Advanced Studies (SOKENDAI), Hayama, Kanagawa 240-0193, Japan. ${ }^{10}$ Nano Life Science Institute (WPI-NanoLSI), Kanazawa University, Kanazawa 920-1192, Japan. These authors contributed equally: Takayuki Uchihashi, Yo-hei Watanabe. Correspondence and requests for materials should be addressed to Y.-h.W. (email: ywatanab@center.konan-u.ac.jp) or to R.I. (email: iino@ims.ac.jp) or to T.A. (email: tando@staff.kanazawa-u.ac.jp)
} 